\title{
Factors associated with choice of first biologic among children with juvenile idiopathic arthritis: a combined analysis from two UK paediatric biologic registers
}

\author{
Rebecca Davies ${ }^{1}$, Lianne Kearsley-Fleet ${ }^{1}$, Eileen Baildam², Michael W. Beresford ${ }^{3}$, Helen Foster ${ }^{4}$, \\ Taunton R. Southwood ${ }^{5}$, Wendey Thomson ${ }^{1 *}$, Kimme L. Hyrich', \\ British Society for Paediatric and Adolescent Rheumatology Etanercept Cohort Study (BSPAR-ETN), \\ Biologics for Children with Rheumatic Diseases Study (BCRD)
}

From 21st European Pediatric Rheumatology (PReS) Congress

Belgrade, Serbia. 17-21 September 2014

\section{Introduction}

The management of juvenile idiopathic arthritis (JIA) has been revolutionised through biologics such as etanercept (ETN), approved in the UK in 2002. Since that time, the use of other biologics in children and young people (CYP) has expanded. ETN is most often the first choice biologic in the treatment of JIA; however there may be occasions where ETN is not the preferred choice, for reasons of efficacy or safety.

\section{Objectives}

The aim of this analysis was to describe the choice of firstline biologics in UK CYP with JIA and explore possible reasons behind this choice.

\section{Methods}

Both the British Society for Paediatric and Adolescent Rheumatology Etanercept Cohort Study (BSPAR-ETN), and the Biologics for Children with Rheumatic Diseases (BCRD) study, are ongoing prospective observational cohorts, collecting detailed information on CYP starting biologics for JIA. At start of therapy, demographic and disease information is collected. Patients registered from 01/01/2010 starting a first biologic were compared between therapies using descriptive statistics. CYP starting ETN <2010 were also included to analyse changes in ETN prescribing since initial approval.

\section{Results}

To 07/04/2014, 870 patients were recruited starting a firstline biologic (123 BCRD; 747 BSPAR-ETN $(582<2010$, $165 \geq 2010$ ) (Table 1). From 2010, CYP with systemic JIA (sIIA) were almost exclusively prescribed anakinra or tocilizumab. Choice of anti-TNF therapy was largely driven by prevalence of uveitis. Compared to ETN patients pre2010, CYP starting ETN from 2010 had shorter disease duration, less uveitis, less sJIA, and less corticosteroid use.

\section{Conclusion}

Although ETN remains the most common biologic prescribed for JIA, there has been a shift towards the use of alternative biologics, some unlicensed, largely driven by disease subtype and the presence of uveitis. This channelling of certain children towards specific therapies is important in terms of future comparative effectiveness studies and also as a guide to ongoing research priorities within rheumatology.

\section{Disclosure of interest}

None declared. 
Table 1

\begin{tabular}{|c|c|c|c|c|c|c|}
\hline $\begin{array}{l}\text { Biologic start post } 01 / 01 / 2010 \text {, unless specified. } \\
{ }^{*} \text { All values median(IQR) or } n(\%)\end{array}$ & $\begin{array}{c}\text { Etanercept } \\
{[\mathrm{N}=165]}\end{array}$ & $\begin{array}{c}\text { Adalimumab } \\
{[\mathrm{N}=45]}\end{array}$ & $\begin{array}{c}\text { Infliximab } \\
{[N=29]}\end{array}$ & $\begin{array}{c}\text { Tocilizumab } \\
{[\mathrm{N}=32]}\end{array}$ & $\begin{array}{c}\text { Anakinra } \\
{[\mathrm{N}=15]}\end{array}$ & $\begin{array}{l}\text { Pre-2010 Etanercept } \\
{[\mathrm{N}=582]}\end{array}$ \\
\hline Female & $109(67 \%)$ & $30(67 \%)$ & 17 (59\%) & $14(44 \%)$ & $11(73 \%)$ & $384(66 \%)$ \\
\hline Age, years & $11(8,14)$ & $10(6,14)$ & $8(5,10)$ & $8(4,11)$ & $3(2,13)$ & $11(8,14)$ \\
\hline Disease duration, years & $2(1,5)$ & $4(2,6)$ & $3(2,6)$ & $1(1,2)$ & $0(0,1)$ & $4(2,7)$ \\
\hline \multicolumn{7}{|l|}{ ILAR Category } \\
\hline Systemic arthritis & $5(3 \%)$ & $1(2 \%)$ & $1(3 \%)$ & $28(88 \%)$ & 15 & 70 (12\%) \\
\hline Oligoarthritis & $39(24 \%)$ & $24(53 \%)$ & $16(55 \%)$ & 0 & $(100 \%)$ & $117(20 \%)$ \\
\hline Polyarthritis & $83(50 \%)$ & $9(20 \%)$ & $9(31 \%)$ & $3(9 \%)$ & 0 & $253(43 \%)$ \\
\hline Enthesitis Related Arthritis & $10(6 \%)$ & $5(11 \%)$ & $2(7 \%)$ & 0 & 0 & $50(9 \%)$ \\
\hline Psoriatic arthritis & $10(6 \%)$ & $5(11 \%)$ & $1(3 \%)$ & 0 & 0 & $44(8 \%)$ \\
\hline \multirow[t]{2}{*}{ Other } & $18(11 \%)$ & $1(2 \%)$ & 0 & $1(3 \%)$ & 0 & $48(8 \%)$ \\
\hline & & & & & 0 & \\
\hline Concomitant MTX & 77 (47\%) & $31(69 \%)$ & $26(90 \%)$ & $28(88 \%)$ & $12(80 \%)$ & $322(55 \%)$ \\
\hline Concomitant corticosteroids & $15(9 \%)$ & $7(16 \%)$ & $5(17 \%)$ & $23(72 \%)$ & $7(47 \%)$ & $146(25 \%)$ \\
\hline Ever had uveitis & $7(5 \%)$ & $31(70 \%)$ & $21(72 \%)$ & 0 & 0 & $54(11 \%)$ \\
\hline CHAQ [0-3] & $1(0,2)$ & $1(0,1)$ & $0(0,1)$ & $1(0,2)$ & $2(1,2)$ & $1(0,2)$ \\
\hline JADAS-71 & $13(8,21)$ & $10(7,17)$ & $6(3,12)$ & $19(1,22)$ & $23(7,30)$ & $16(9,23)$ \\
\hline
\end{tabular}

\section{Authors' details}

'Arthritis Research UK Centre for Epidemiology, Manchester Academic Health

Science Centre, The University of Manchester, Manchester, UK. ${ }^{2}$ Hey

Children's Foundation NHS Trust, Liverpool, UK. Institue of Translational

Medicine (Child Health), University of Liverpool, Liverpool, UK

${ }^{4}$ Musculoskeletal Research Group, Institute Cellular Medicine, Newcastle

University, Newcastle upon Tyne, UK. ${ }^{5}$ Department of Paediatric

Rheumatology, Institute of Child Health, Birmingham Children's Hospital -

NHS Trust and University of Birmingham, Birmingham, UK.

Published: 17 September 2014

doi:10.1186/1546-0096-12-S1-P190

Cite this article as: Davies et al:: Factors associated with choice of first biologic among children with juvenile idiopathic arthritis: a combined analysis from two UK paediatric biologic registers. Pediatric

Rheumatology 2014 12(Suppl 1):P190.

\section{Submit your next manuscript to BioMed Central and take full advantage of:}

- Convenient online submission

- Thorough peer review

- No space constraints or color figure charges

- Immediate publication on acceptance

- Inclusion in PubMed, CAS, Scopus and Google Scholar

- Research which is freely available for redistribution

Submit your manuscript at www.biomedcentral.com/submit
C Biomed Central 